\title{
Mass Accretion Impacts in Classical T Tauri Stars: A Multi-disciplinary Approach
}

\author{
S. Orlando, C. Argiroffi, R. Bonito, S. Colombo, G. Peres, F. Reale, M. Miceli, \\ L. Ibgui, C. Stehlé, and T. Matsakos
}

\section{Introduction}

According to the magnetospheric accretion scenario, young low-mass stars are surrounded by circumstellar disks with which they interact in a complex fashion, with accretion of mass and ejection of collimated outflows. The accretion process is regulated by the stellar magnetic field which disrupts the inner part of the disk at a distance of a few stellar radii (the truncation radius) and guides the disk's material toward the central star. The impact of this material onto the stellar surface is expected to generate a shock which propagates through the accretion column and heats the downfalling material up to temperatures of million degrees. The impacts, therefore, are expected to generate X-ray emission.

S. Orlando $(\varangle) \cdot$ R. Bonito

INAF - Osservatorio Astronomico di Palermo, Palermo, Italy

e-mail: salvatore.orlando@inaf.it; rosaria.bonito@inaf.it

C. Argiroffi · G. Peres · F. Reale · M. Miceli

Dip. di Fisica e Chimica, Università di Palermo, Sicily, Italy

INAF - Osservatorio Astronomico di Palermo, Palermo, Italy

e-mail: costanza.argiroffi@unipa.it; giovanni.peres@unipa.it; fabio.reale@unipa.it; marco.miceli@unipa.it

S. Colombo

INAF - Osservatorio Astronomico di Palermo, Palermo, Italy

LERMA, Sorbonne Université, Observatoire de Paris, Université PSL, CNRS, Paris, France e-mail: salvatore.colombo@inaf.it

L. Ibgui · C. Stehlé · T. Matsakos

LERMA, Sorbonne Université, Observatoire de Paris, Université PSL, CNRS, Paris, France e-mail: laurent.ibgui@obspm.fr; chantal.stehle@obspm.fr; titos.matsakos@obspm.fr 
In fact, high resolution X-ray observations of young stars accreting material from their circumstellar disks (e.g. TW Hya, BP Tau, V4046 Sgr, MP Mus and RU Lupi) have revealed $\mathrm{X}$-ray emission from plasma at $T \approx 2-5 \mathrm{MK}$, which is denser than $n_{H}=10^{11} \mathrm{~cm}^{-3}$ [9]. This soft X-ray emission component could be produced by the material accreting onto the star surface, flowing along the magnetic field lines of the nearly dipolar stellar magnetosphere, and heated to temperatures of few MK by a shock at the base of the accretion column [3, 11].

In the last years several models have been proposed to describe the impacts of accreting material onto the surface of classical T Tauri stars (CTTSs). They provide a convincing theoretical support and a plausible global picture of the phenomenon at work. However some fundamental aspects of accretion impacts still need to be clarified: the observed X-ray luminosity produced by plasma heated in accretion shocks is largely below the predicted value [6]; the observed coronal activity seems to be influenced by accretion but it is not clear why and how $[8,14]$; UV emission lines exhibit complex profiles and large doppler shifts [1]. In this contribution, we briefly review some of the achievements obtained by our group by exploiting a multi-disciplinary approach based on the analysis of multi-dimensional magnetohydrodynamic simulations, multi-wavelength observations, and laboratory experiments of accretion impacts occurring onto the surface of CTTSs.

\section{Structure and Evolution of Shock-Heated Plasma in Accretion Impacts}

The first numerical models describing the impact of an accretion column onto the surface of a CTTS were one-dimensional (1D) and they have shown that the continuous impact of an accretion column onto the stellar surface leads to the formation of a dense and hot slab of plasma that undergoes sandpile quasiperiodic (QPOs) oscillations driven by catastrophic cooling [10, 19, 20]. The major achievement of these models has been to demonstrate that the main features of high spectral resolution X-ray observations of CTTSs can be reproduced by accretion impacts and originate from post-shock plasma [19]. These models however are justified if the plasma has a $\beta \ll 1$ (where $\beta=$ gas pressure / magnetic pressure) in the shock-heated material: in this conditions the plasma is assumed to move and to transport energy only along magnetic field lines.

The stability and dynamics of accretion impacts in cases where the low- $\beta$ approximation cannot be applied have been investigated through two-dimensional (2D) magnetohydrodynamic (MHD) models [4, 12, 13, 15]. These models have shown that, in general, QPOs cannot be observed (according to the absence of periodic X-ray modulation due to shock oscillations found in observations; [7]) due to heavy dumping by the magnetic field [13] or to perturbation of the stream induced by the post-shock plasma itself $[4,12,15]$. The atmosphere around the 
impact region of the stream can be strongly perturbed (depending on the plasma $\beta$ ), leading to important leaks at the border of the main stream [15].

The 2D MHD models have also shown that the strength and configuration of the magnetic field play a crucial role in determining the dynamics and evolution of the post-shock plasma. In the case of weak magnetic fields (plasma $\beta \gtrsim 1$ in the postshock region), a large component of $\mathbf{B}$ may develop perpendicular to the stream at the base of the accretion column. This component limits the sinking of the shocked plasma into the chromosphere $[13,15]$. For strong magnetic fields $(\beta<1$ in the post-shock region close to the chromosphere), the field configuration determines the position of the shock and its stand-off height [13]. If the field is strongly tapered close to the chromosphere, an oblique shock may form well above the stellar surface at the height where the plasma $\beta \approx 1$.

The above models have allowed to investigate the structure, stability, and location of the post-shock plasma. However they do not explain the evidence that a significant amount of plasma at $10^{5} \mathrm{~K}$ is produced in the accretion process. Also they are not able to reproduce UV observations which show the C IV doublet at $1550 \AA$ with a complex profile described by two Gaussian components with different speeds and widths [1]: a narrow component redshifted at speeds of $\approx 30 \mathrm{~km} \mathrm{~s}^{-1}$ and a broader component centered at $\approx 120 \mathrm{~km} \mathrm{~s}^{-1}$ and with the redshifted wing reaching $\approx 400 \mathrm{~km} \mathrm{~s}^{-1}$.

Surprisingly solar observations suggested a way to explain the origin of the observed asymmetries and redshifts of UV emission lines in CTTSs. After a violent eruption occurred on 2011 June 7, dense plasma fragments were observed to fall back on the surface of the Sun, producing brightenings in X-rays after the impacts. These impacts have been shown to reproduce on the small scale accretion impacts onto CTTSs [17]. The hydrodynamic modeling of the impacts observed in the Sun and the synthesis of emission in UV and X-ray bands from the models have shown that UV emission may originate from the shocked front shell of the still downfalling fragments [16]. In this case a broad redshifted component in UV lines is produced up to speeds around $\approx 400 \mathrm{~km} \mathrm{~s}^{-1}$ which is consistent with UV observations of CTTSs.

The hypothesis that the accreting material can be fragmented or clumpy has been challenged by an MHD model which describes an accretion column consisting of several high density blobs which impact onto the chromosphere of a CTTS [4]. The model has shown that the impacts of the blobs produce shocked upflows which, possibly, hit and shock the subsequent downfalling fragments. As a result, several shocked plasma components with different downfalling velocities are present altogether, leading to profiles of C IV lines remarkably similar to those observed in CTTSs. The fragmentation of the accreting material, therefore, may explain in a natural way the origin of asymmetries and redshifts of UV emission lines in CTTSs. 


\section{Effect of Optically Thick Plasma Around Impact Regions}

The analysis of observations in different bands has shown the evidence that the mass accretion rates derived from X-rays are significantly lower than those derived from other spectral bands (UV/optical/NIR observations; [6]). Early 1D models of accretion impacts have suggested a reason for this discrepancy. They pointed out the importance of absorption from the optically thick material of the chromosphere in the post-shock plasma components that produce observable emission in the X-ray band [20]. In addition 2D MHD models have shown that absorption of Xray emission may originate from the presence of optically thick material located around the impact regions. In the case of plasma $\beta \gtrsim_{1}$ in the post-shock region, an envelope of dense and cold chromospheric material may develop around the shocked column. This envelope is expected to determine a significant absorption of the X-ray emission arising from the post-shock plasma [13].

The synthesis of X-ray emission from 2D MHD models has shown that, if the effects of local absorption are taken into account, the X-ray fluxes inferred from the emerging spectra are lower than expected because of the complex local absorption by the optically thick material of the chromosphere and of the unperturbed stream [2]. The first attempt to investigate in more detail the effects of radiative transfer during accretion impacts in CTTSs was performed through a 1D hydrodynamic model [5]. This model has shown that the dense and cold plasma of the pre-shock accretion column is gradually heated up to a few $10^{5} \mathrm{~K}$ due to irradiation of $\mathrm{X}$ rays arising from the shocked plasma at the impact region. As a result, a region of radiatively heated gas (a precursor) forms in the unshocked accretion column and contributes significantly to UV emission. In such a way, the model naturally reproduces the luminosity of UV emission lines correlated to accretion and shows that most of the UV emission originates from the precursor.

The hypothesis of absorption of X-ray emission in accretion impacts has been strongly supported by the analysis of solar observations and laboratory experiments. Observations of bright hot impacts by erupted fragments falling back on the Sun have shown that the X-ray emission produced in the impacts is heavily absorbed by optically thick plasma, possibly explaining the discrepancy between mass accretion rates derived from X-ray and optical observations [17]. Scaled laboratory experiments of collimated plasma accretion onto a solid in the presence of a magnetic field were able to track, with spatial and temporal resolution, the dynamics of the system and simultaneously measured multi-band emissions [18]. The experiments have shown that optically thick material due to the perturbation of the chromosphere may envelope the impact region and determine a partial absorption of the X-ray emission. Once again, this finding supports absorption as possible reason reconciling current discrepancies between mass accretion rates derived from X-ray and optical observations, respectively. 


\section{Summary and Conclusions}

We summarized some of the recent achievements in the study of accretion impacts in CTTSs obtained by adopting a multi-disciplinary approach. The MHD models provide a plausible global picture of the phenomenon at work. They were able to investigate the effects of stellar magnetic field in determining the structure, stability, and location of the shocked plasma. In particular they have suggested that the distribution of dense and cold material around the hot slab may lead to significant absorption of X-ray emission arising from the post-shock plasma. The structure of the hot slab can be largely affected by chromospheric or stochastic flow effects. The analysis of observability of accretion shocks in UV and X-ray bands has shown that the observed asymmetric and redshifted line profiles can be explained by fragmented or clumpy accretion columns. The laboratory experiments can provide important complementary information on the physics of accretion impacts. For instance, it was possible to prove that heavy absorption of X-ray emission by optically thick material is present around impact regions. The evidence of absorption of X-ray emission may reconcile current discrepancies between mass accretion rates derived from X-ray and optical observations. Finally, it was proved that solar observations can provide a powerful template for stellar accretion. Thanks to these observations it was possible to prove the importance of local absorption in suppressing the X-ray emission from the post-shock plasma and to find a way to explain the origin of the observed asymmetries and redshifts of UV emission lines in CTTSs.

Acknowledgements The PLUTO code, used in this work, is developed at the Turin Astronomical Observatory in collaboration with the Department of General Physics of Turin University and the SCAI Department of CINECA. These studies have been partially funded by the French Italian cooperation program PICS 6838 "Physics of Mass Accretion Processes in Young Stellar Objects", and the LABEX PLAS@PAR (ANR-11-IDEX-0004-02).

\section{References}

1. Ardila, D.R., Herczeg, G.J., Gregory, S.G., Ingleby, L., France, K., Brown, A., Edwards, S., Johns-Krull, C., Linsky, J.L., Yang, H., Valenti, J.A., Abgrall, H., Alexander, R.D., Bergin, E., Bethell, T., Brown, J.M., Calvet, N., Espaillat, C., Hillenbrand, L.A., Hussain, G., Roueff, E., Schindhelm, E.R., Walter, F.M. 2013, ApJS, 207, 1

2. Bonito, R., Orlando, S., Argiroffi, C., Miceli, M., Peres, G., Matsakos, T., Stehle, C., Ibgui, L. 2014, ApJ, 795, L34

3. Calvet, N., Gullbring, E. 1998, ApJ, 509, 802

4. Colombo, S., Orlando, S., Peres, G., Argiroffi, C., Reale, F. 2016, A\&A, 594, A93

5. Costa, G., Orlando, S., Peres, G., Argiroffi, C., Bonito, R. 2017, A\&A, 597, A1

6. Curran, R.L., Argiroffi, C., Sacco, G.G., Orlando, S., Peres, G., Reale, F., Maggio, A. 2011, A\&A, 526, A104

7. Drake, J.J., Ratzlaff, P.W., Laming, J.M., Raymond, J. 2009, ApJ, 703, 1224

8. Flaccomio, E., Damiani, F., Micela, G., Sciortino, S., Harnden Jr., F.R., Murray, S.S., Wolk, S.J. 2003, ApJ, 582, 398 
9. Kastner, J.H., Huenemoerder, D.P., Schulz, N.S., Canizares, C.R., Weintraub, D.A. 2002, ApJ, 567,434

10. Koldoba, A.V., Ustyugova, G.V., Romanova, M.M., Lovelace, R.V.E. 2008, MNRAS, 388, 357

11. Lamzin, S.A. 1998, Astronomy Reports, 42, 322

12. Matsakos, T., Chièze, J.P., Stehlé, C., González, M., Ibgui, L., de Sá, L., Lanz, T., Orlando, S., Bonito, R., Argiroffi, C., Reale, F., Peres, G. 2013, A\&A, 557, A69

13. Orlando, S., Bonito, R., Argiroffi, C., Reale, F., Peres, G., Miceli, M., Matsakos, T., Stehlé, C., Ibgui, L., de Sa, L., Chièze, J.P., Lanz, T. 2013, A\&A, 559, A127

14. Orlando, S., Reale, F., Peres, G., Mignone, A. 2011, MNRAS, 415, 3380

15. Orlando, S., Sacco, G.G., Argiroffi, C., Reale, F., Peres, G., Maggio, A. 2010, A\&A, 510, A71

16. Reale, F., Orlando, S., Testa, P., Landi, E., Schrijver, C.J. 2014, ApJ, 797, L5

17. Reale, F., Orlando, S., Testa, P., Peres, G., Landi, E., Schrijver, C.J. 2013, Science, 341, 251

18. Revet, G., Chen, S.N., Bonito, R., Khiar, B., Filippov, E., Argiroffi, C., Higginson, D.P., Orlando, S., Béard, J., Blecher, M., Borghesi, M., Burdonov, K., Khaghani, D., Naughton, K., Pépin, H., Portugall, O., Riquier, R., Rodriguez, R., Ryazantsev, S.N., Skobelev, I.Y., Soloviev, A., Willi, O., Pikuz, S., Ciardi, A., Fuchs, J. 2017, Science Advances, 3, e1700982

19. Sacco, G.G., Argiroffi, C., Orlando, S., Maggio, A., Peres, G., Reale, F. 2008, A\&A, 491, L17

20. Sacco, G.G., Orlando, S., Argiroffi, C., Maggio, A., Peres, G., Reale, F., Curran, R.L. 2010, A\&A, 522, A55 